- promotion: that future health care planning and end of life discussions are normalised in society

- resources: ACP is accessible to all regardless of language, literacy level or cultural beliefs

- education and training: we have workforce and community prepared to have conversations and use ACPs

- monitoring and evaluation: we know care is based on what matters to consumers

- implementation: we are maximising value for DHBs

Result a national mandate, strategy and specified actions have resulted in an increase in ACP activity; increased governance with decision-making being supported by a representative Steering Group; wider national stakeholder engagement and buy in from agencies in and outside of the health sector.

\section{OP80 SHARED CARE PLANNING: A NEW MODEL TO INTEGRATE ADVANCE CARE PLANNING INTO COMMUNITY. THE BASQUE COUNTRY EXPERIENCE}

I Saralegui*. Organización Sanitaria Integrada Araba, Vitoria-Gasteiz, Spain

\subsection{6/spcare-2019-ACPICONGRESSABS.80}

In the Basque Country, a project has been implemented based on community education, the training of health and social workers. It tries to elicit the individual's preferences and encourages them to take part in planning their care. Writing down advance directives is not the main aim.

The target population is chronic patients, elderly people and anyone at the end of life. The project also includes everyone who wants to think about the process of dying and needs to have a conversation related to this topic.

The project started in 2014 as a bottom-up project and it has grown into a top-down project performed in the whole Basque Health Service.

Results More than one hundred conferences and debates have been taking place in neighborhood associations, cultural centers, libraries or educational centers. More than five thousand people have attended these activities. One thousand workers have attended a basic training course, more than 500 workers have been trained as SCP facilitators and more than 700 doctors and nurses have participated in conversations with patients and families helped by an SCP facilitator. In $75 \%$ of cases, the citizens asked to be included after attending a conference; only $25 \%$ of participants were included because of a doctor or nurse's invitation. Many support documents have been created in order to explain the project and to make it easier to understand.

The keystone is the training of health and social workers in order to answer citizens' requests and to integrate the conversations into everyday care.

\section{OP81 IMPLEMENTATION OF ADVANCE CARE PLANNING IN AGED CARE: WHAT DO YOU WANT AND HOW CAN WE HELP?}

${ }^{1} \mathrm{H}$ Kelly, ${ }^{1} \mathrm{~L}$ Nolte, ${ }^{2} \mathrm{M}$ Fearn, ${ }^{2} \mathrm{~F}$ Batchelor, ${ }^{2} \mathrm{~B}$ Haralambous, ${ }^{2} \mathrm{P}$ Mackell, ${ }^{2} \mathrm{~K}$ Hwang, ${ }^{1} \mathrm{~K}$ Detering. ${ }^{1}$ Advance Care Planning Australia, Melbourne, Australia; ${ }^{2}$ National Ageing Research Institute Inc, Melbourne, Australia

10.1136/spcare-2019-ACPICONGRESSABS.81
Introduction The goal of advance care planning (acp) is to ensure that individuals receive future care consistent with their expressed preferences. Benefits of acp include: increased adherence to a person's preferences, higher staff satisfaction, reductions in unwanted hospitalisations and medical treatments, and reduced stress and anxiety for family. Despite clear benefits, uptake of acp has been limited in Australian aged care settings. This research explored barriers and enablers to implementing acp in residential and community aged care settings, from the perspectives of aged care staff and older people.

Method Focus groups and interviews with aged care staff and older people were conducted in four Australian states. Sixty staff from 15 residential and community aged care organisations and 24 older people participated. A thematic analysis was undertaken to identify facilitators and barriers.

Results Factors identified as impacting on the experiences and implementation of acp included: knowledge and understanding access to education/training understanding of relevant legislation having defined roles/responsibilities cognitive capacity of the older person timing of initiating the conversation availability of clear policies/procedures engaging older people and their families diversity within the workforce and older people.

Conclusions Aged care staff indicated there are a range of factors that can impact on acp implementation. All these factors may need to be considered when implementing acp within Australian aged care organisations. The findings informed the development of a new national resource titled Advance care planning in aged care: a guide to support implementation in community and residential settings.

\section{OP82 IDENTIFICATION, IMPLEMENTATION AND EVALUATION OF INDICATORS TO MONITOR SUCCESSFUL UPTAKE OF ADVANCE CARE PLANNING IN ALBERTA}

${ }^{1,2} \mathrm{~J}_{\mathrm{Xiao}}{ }^{*}{ }^{3} \mathrm{~J}$ SimOn, ${ }^{4} \mathrm{~T}$ Lynn Wityk Martin, ${ }^{4} \mathrm{~S}$ Iversen, ${ }^{1} \mathrm{M}$ Douglas, ${ }^{1} \mathrm{~A}$ Potapov, ${ }^{1} \mathrm{M}$ Nesari, ${ }^{3} \mathrm{P}$ Biondo, ${ }^{3} \mathrm{~A}$ Kanters, ${ }^{1,2} \mathrm{~K}$ Fassbender. ${ }^{1}$ University of Alberta, Edmonton, Canada; ${ }^{2}$ Covenant Health Palliative Institute, Edmonton, $A B$, Canada; ${ }^{3}$ University of Calgary, Calgary, Canada; ${ }^{4}$ Alberta Health Services, Edmonton, $A B$, Canada

\subsection{6/spcare-2019-ACPICONGRESSABS.82}

Background In 2014, a province-wide policy for advance care planning (ACP) and Goals of Care Designation (GCD) was implemented in Alberta, Canada; nevertheless, few quality indicators have been rigorously developed or evaluated for measuring the uptake of ACP/GCD.

Methods In phase I, we performed a systematic literature review and environmental scan to identify potential ACP/GCD indicators. A Delphi consensus-based approach, consisting of 3 rounds of face-to-face meetings and/or online surveys, was used to develop a short list of indicators. In phase II, the panelists met face-to-face to operationalize and implement the indicators. In phase III, two validated questionnaires and semistructured interviews of 60 individuals (stratified by manager/ practitioner and physician/nurse) are being used to evaluate the usability and acceptability of the implemented indicators on a dashboard interface.

Results A total of 132 potential indicators were identified in phase I. The indicators were reduced and refined to 18 after 3 Delphi rounds. Phase II resulted in 9 valid and feasible indicators in a measurable format (i.e. numerator, denominator, data source defined). The Phase III protocol is under 